\title{
GMR
}

\section{Correlation between magnetic resonance perfusion weighted imaging of radiation brain injury and pathology}

\author{
X.J. Liu, C.F. Duan, W.W. Fu, L. Niu, Y. Li, Q.L. Sui and W.J. Xu \\ Department of Radiology, Affiliated Hospital of Qingdao University, Qingdao, China \\ Corresponding authors: Q.L. Sui / W.J. Xu \\ E-mail: suiqinglaa_|@163.com / xuwenjian_II@163.com
}

Genet. Mol. Res. 14 (4): 16317-16324 (2015)

Received July 3, 2015

Accepted September 18, 2015

Published December 8, 2015

DOI http://dx.doi.org/10.4238/2015.December.8.23

\begin{abstract}
We used magnetic resonance perfusion weighted imaging and pathological evaluation to examine different stages of radiation-induced brain injury and to investigate the correlation between the relative cerebral blood volume (rCBV) ratio and vascular endothelial growth factor (VEGF). Thirty adult rats were randomly divided into 2 groups: control and radiation group. The control group was not subjected to irradiation. The irradiation group rats were examined by magnetic resonance imaging and magnetic resonance perfusion weighted imaging at 1, 3, 6, 9, and 12 months after radiation treatment. We measured the $\mathrm{CCBV}$, mean transit time, and time to peak. Hematoxylin and eosin staining, immunohistochemical staining, and electron microscopy were performed. VEGF absorbance was evaluated by immunohistochemical staining. Compared with the control group, the differences in rCBV, mean transit time, time to peak, and VEGF absorbance after 3 months were statistically significant $(P<0.05)$. rCBV was positively correlated with VEGF $(r=0.94, P<0.05)$. Magnetic resonance perfusion weighted imaging can reflect pathophysiological changes in brain tissue
\end{abstract}


after irradiation. Decreased expression of VEGF plays a critical role in the pathogenesis of radiation-induced brain injury.

Key words: Magnetic resonance perfusion weighted imaging; Radiation-induced brain injury; Vascular endothelial growth factor; Immunohistochemistry

\section{INTRODUCTION}

Brain radiation injury is a severe complication of radiotherapy, with atypically clinical symptoms during early stages. Conventional imaging is insensitive and leads to irreversible brain injury after symptoms are detected (Tsao et al., 2005; Asai and Kawamoto, 2008). Therefore, the early diagnosis of radiation brain injury is very important. We induced brain radiation injury in rats and evaluated these injuries using magnetic resonance perfusion weighted imaging (MRP) and pathological examination for early diagnosis.

\section{MATERIAL AND METHODS}

\section{Establishment of brain radiation injury in rats}

Thirty female Wistar rats weighing 200-230 g were obtained from Qingdao Medicine Institute and randomly divided into a control group and 1, 3, 6, 9, and 12 months after radiation groups, with 5 rats in each group.

Intraperitoneal anesthesia was performed using $10 \%$ chloral hydrate with $0.35 \mathrm{~mL} / 100 \mathrm{~g}$, and then subjected to single-whole-vertical brain irradiation with a 6-MeV electron beam using a 23EX linear accelerator (Varian, Palo Alto, CA, USA), $300 \mathrm{~Gy} / \mathrm{min}, 3.0 \times 20 \mathrm{~cm}, 100 \mathrm{~cm}$ source-skin distance, $1.5-\mathrm{cm}$ reference dose depth, and total dose of $20 \mathrm{~Gy}$.

\section{Magnetic resonance examination}

We used an HDX 3.0T MRI scanner (GE Healthcare, Little Chalfont, UK) and a phased array rat coil from Shanghai Chenguang Medical Technologies Co., Ltd. (CG-MVC22-H300-AG; Shanghai, China).

The following parameters were used: $\mathrm{T} 1$ weighted image $(\mathrm{T} 1 \mathrm{WI})$ repetition time $(\mathrm{TR})=$ $400 \mathrm{~ms}$, echo time $(T E)=11 \mathrm{~ms}$, field-of-view $(F O V)=8 \mathrm{~cm} \times 8 \mathrm{~cm}$, slice thickness $=2.4 \mathrm{~mm}$, interlayer distance $=0 \mathrm{~mm}$. T2WI TR $=3500 \mathrm{~ms}, \mathrm{TE}=110 \mathrm{~ms}, \mathrm{FOV}=8 \times 8 \mathrm{~cm}$, slice thickness $=$ $2.4 \mathrm{~mm}$, interlayer distance $=0 \mathrm{~mm}$. For perfusion imaging, the following parameters were used: $\mathrm{TR}=1000 \mathrm{~ms}, \mathrm{TE}=15.9 \mathrm{~ms}, \mathrm{FOV}=8 \mathrm{~cm} \times 6 \mathrm{~cm}$, flip angle $=30^{\circ}$, matrix $=64 \times 64$, slice thickness $=2.4 \mathrm{~mm}$, interlayer distance $=0 \mathrm{~mm}$. The echo planar sequence was acquired using MRP for a total of 100 times, with 10 layers in each scanning. After the 4th scanning, a needle was placed in the femoral vein and $0.1 \mathrm{mmol} / \mathrm{kg}$ Gadolinium was injected at a rate of $1 \mathrm{~mL} / \mathrm{s}$, while $2 \mathrm{~mL} / \mathrm{kg}$ normal saline () was injected at the same rate into control rats. For magnetic resonance enhanced scanning, the following parameters were used: TR $=1025 \mathrm{~ms}, \mathrm{TE}=24 \mathrm{~ms}, \mathrm{TI}=860 \mathrm{~ms}, \mathrm{FOV}=8$ $\mathrm{x} 6 \mathrm{~cm}$, slice thickness $=2.4 \mathrm{~mm}$, interlayer distance $=0 \mathrm{~mm}$. 


\section{Pathological examination}

Rats were examined by magnetic resonance, and then the brains were removed immediately, placed under normal saline lavage, and fixed with $4 \%$ paraformaldehyde. The brain tissues of rats were dehydrated in an ascending gradient series of ethanol, cleared in xylene, and embedded in paraffin. Tissue slices were prepared and subjected to hematoxylin and eosin staining and vascular endothelial growth factor (VEGF) staining.

\section{Figures and data analysis}

Data were analyzed using Functool software. MRP perfusional color maps were obtained and treated. Six randomly selected regions with the same area (region of interest $=16-20 \mathrm{~mm}^{2}$ ) were examined to detect the relative cerebral blood volume (rCBV), mean transit time (MTT), and time to peak (TTP), and average values were calculated.

The absorbance value of VEGF was determined using Image-pro plus 5.0 as the average of 3 measurements. Hematoxylin and eosin staining was used to determine the morphology, structure, density, and small structure changes of the vascular lumen and basement membrane under an electron microscope.

\section{Statistical analysis}

Data were analyzed using SPSS 17.0 (SPSS, Inc., Chicago, IL, USA). Values are reported as means \pm SD. Groups were compared by one-way analysis of variance. Measurement data were compared using the least square differences- $t$ test, while the Dunnett and T3 tests were used to detect variance non-homogeneity. A value of $P<0.05$ was considered to indicate a significant different. Correlations between rCBV and VEGF were represented as $r$, which is the product moment correlation coefficient for the Pearson test, and examined using the $t$-test. A value of $\mathrm{P}<$ 0.05 was considered to indicate statistical significance.

\section{RESULTS}

\section{MRP results}

rCBV in each radiation group decreased gradually over time. There were significant differences between the 3-, 6-, 9-, and 12-month groups and the control group (Table 1 and Figure 1a-c).

\begin{tabular}{|c|c|c|c|c|c|c|}
\hline \multirow[t]{2}{*}{ Groups } & \multirow[t]{2}{*}{ Means \pm SD } & \multicolumn{3}{|c|}{ Intra-group (vs control) } & \multicolumn{2}{|c|}{ Between groups } \\
\hline & & Mean difference & Standard error & $\mathrm{P}$ & $\mathrm{F}$ & $P$ \\
\hline Control & $288 \pm 68$ & & & & & \\
\hline 1 month & $325 \pm 33$ & -36.2 & 28.675 & $>0.05$ & & \\
\hline 3 months & $233 \pm 28$ & 55.2 & 28.675 & $<0.05$ & & \\
\hline 6 months & $213 \pm 53$ & 75.0 & 28.675 & $<0.05$ & & \\
\hline 9 months & $175 \pm 45$ & 113.6 & 28.675 & $<0.05$ & & \\
\hline 12 months & $152 \pm 28$ & 136.6 & 28.675 & $<0.05$ & 10.60 & $<0.05$ \\
\hline
\end{tabular}



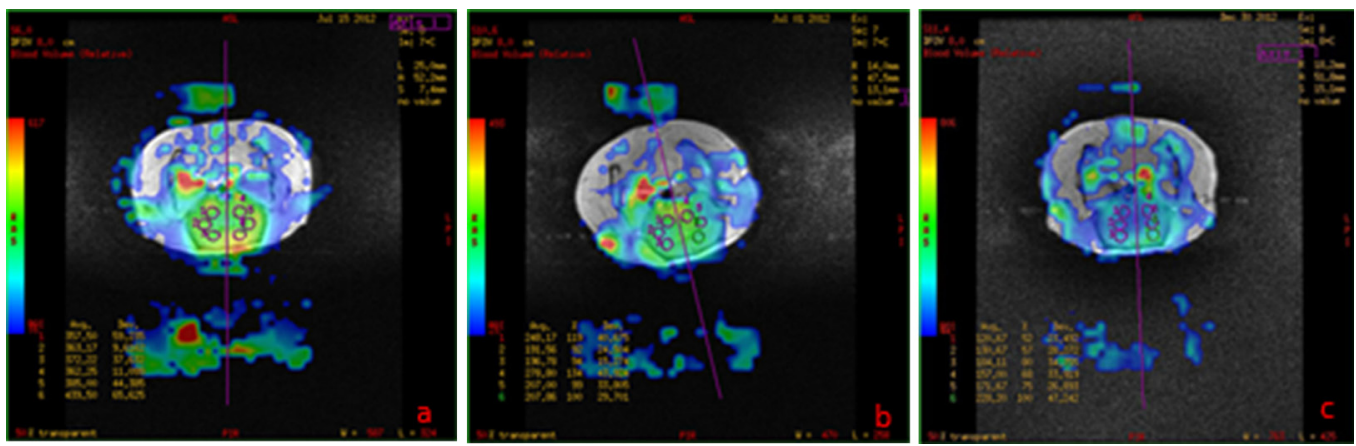

Figure 1. rCBV color maps of the control group and radiation groups: control (a), 3 months (b), and 12 months (c). Overtime, rCBV decreased and reached the lowest level at 12 months, while the color maps changed from yellowish green to blue.

Table 2 shows that MTT clearly increased gradually over time. There were significant differences between the 3-, 6-, 9-, and 12-month groups and the control group.

Table 2. MTT value and comparison between groups (one-way analysis of variance), intra-groups (least square differences - $t$-test).

\begin{tabular}{lcccccc}
\hline Groups & Means \pm SD & \multicolumn{3}{c}{ Intra-group (vs control) } & & \multicolumn{2}{c}{ Between groups } \\
\cline { 3 - 4 } & & Mean difference & Standard error & $\mathrm{P}$ & & $\mathrm{F}$ \\
\hline Control & $3.98 \pm 0.68$ & & & & \\
1 month & $4.08 \pm 0.46$ & -0.1 & 1.169 & $>0.05$ & \\
3 months & $6.70 \pm 0.92$ & -2.712 & 1.169 & $<0.05$ & \\
6 months & $6.65 \pm 2.06$ & -2.462 & 1.169 & $<0.05$ & \\
9 months & $9.62 \pm 2.36$ & -5.636 & 1.169 & $<0.05$ & \\
12 months & $11.75 \pm 3.02$ & -7.764 & 1.169 & $<0.05$ & 13.8 & $<0.05$ \\
\hline
\end{tabular}

Table 3 shows that TPP clearly increased gradually over time. There were significant differences between the 3-, 6-, 9-, and 12-month groups and the control group.

Table 3. TPP value and comparison between groups (one-way analysis of variance), intra-groups (least square differences - $t$-test).

\begin{tabular}{|c|c|c|c|c|c|c|}
\hline \multirow[t]{2}{*}{ Groups } & \multirow[t]{2}{*}{ Means \pm SD } & \multicolumn{3}{|c|}{ Intra-group (vs control) } & \multicolumn{2}{|c|}{ Between groups } \\
\hline & & Mean difference & Standard error & $\mathrm{P}$ & $\mathrm{F}$ & $P$ \\
\hline Control & $6.85 \pm 2.23$ & & & & & \\
\hline 1 month & $8.79 \pm 1.01$ & -1.934 & 4.347 & $>0.05$ & & \\
\hline 3 months & $17.31 \pm 3.90$ & -10.454 & 4.347 & $<0.05$ & & \\
\hline 6 months & $16.70 \pm 2.91$ & -10.144 & 4.347 & $<0.05$ & & \\
\hline 9 months & $24.89 \pm 10.11$ & -18.036 & 4.347 & $<0.05$ & & \\
\hline 12 months & $25.21 \pm 12.31$ & -18.354 & 4.347 & $<0.05$ & 6.34 & $<0.05$ \\
\hline
\end{tabular}

\section{Pathological examination}

Table 4 shows that VEGF clearly decreased gradually over time. There were significant differences between the 3-, 6-, 9-, and 12-month groups and the control group.

Light microscopy analysis showed that in the control group, the vascular density was 
normal and well-distributed. The vascular wall had a uniform thickness. The vessel lumen was smooth and regular. After 3 months, vascular density in the radiation groups decreased gradually, the vascular wall thickened slightly, and the vessel lumen became slightly narrower. Over time, these changes became more pronounced. Uptil 12 months, vascular density decreased, the vascular wall thickened, and the vessel lumen became narrower or blocked (Figure 2). Electron microscopy analysis revealed that in the control group, vascular density was normal and welldistributed. A number of endothelial cells were observed and these cells had complete structures. Tight junctions were observed between the cells. Over time, the basement membrane thickened gradually, endothelial cells underwent apoptosis, and the cell number decreased. Until the 12th month, the number of endothelial cells decreased, the tight junctions gradually disappeared, and organelle structures, including the mitochondria and endoplasmic reticulum, dissolved and disappeared (Figure 3).

Table 4. Absorbance value of VEGF and comparison between groups (one-way analysis of variance), intragroups (least square differences $-t$-test).

\begin{tabular}{|c|c|c|c|c|c|c|}
\hline \multirow[t]{2}{*}{ Groups } & \multirow[t]{2}{*}{ Means \pm SD } & \multicolumn{3}{|c|}{ Intra-group (vs control) } & \multicolumn{2}{|c|}{ Between groups } \\
\hline & & Mean difference & Standard error & $\mathrm{P}$ & $\mathrm{F}$ & $P$ \\
\hline Control & $1.178 \pm 0.087$ & & & & & \\
\hline 1 month & $1.167 \pm 0.110$ & 0.011 & 0.395 & $>0.05$ & & \\
\hline 3 months & $1.003 \pm 0.075$ & 0.175 & 0.395 & $<0.05$ & & \\
\hline 6 months & $0.781 \pm 0.044$ & 0.397 & 0.395 & $<0.05$ & & \\
\hline 9 months & $0.731 \pm 0.037$ & 0.447 & 0.395 & $<0.05$ & & \\
\hline 12 months & $0.582 \pm 0.022$ & 0.596 & 0.395 & $<0.05$ & 77.56 & $<0.05$ \\
\hline
\end{tabular}

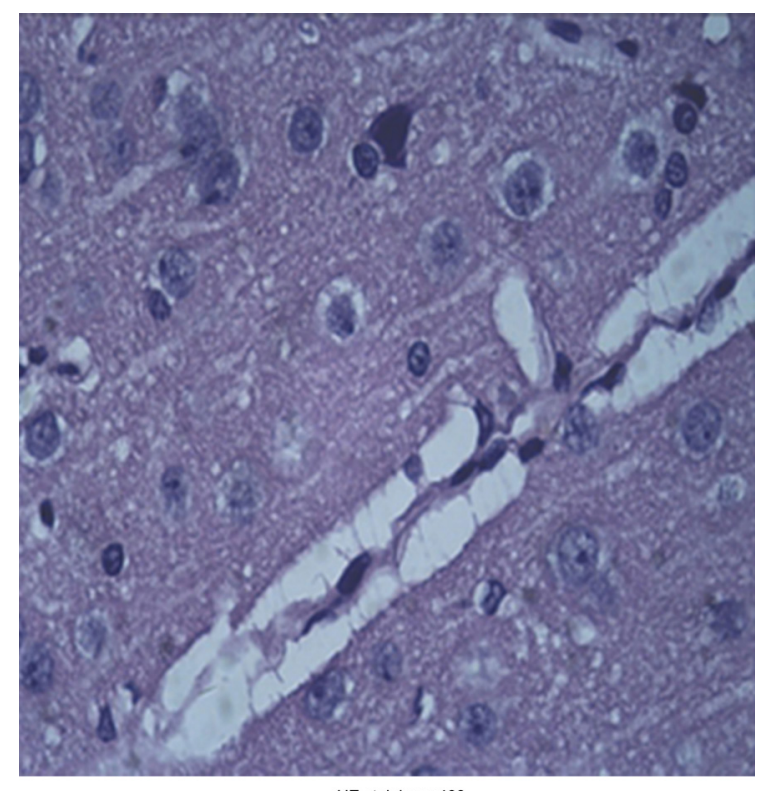

HE staining $\times 400$

Figure 2. Vascular wall thickened and the lumen became blocked during the 12th month. 


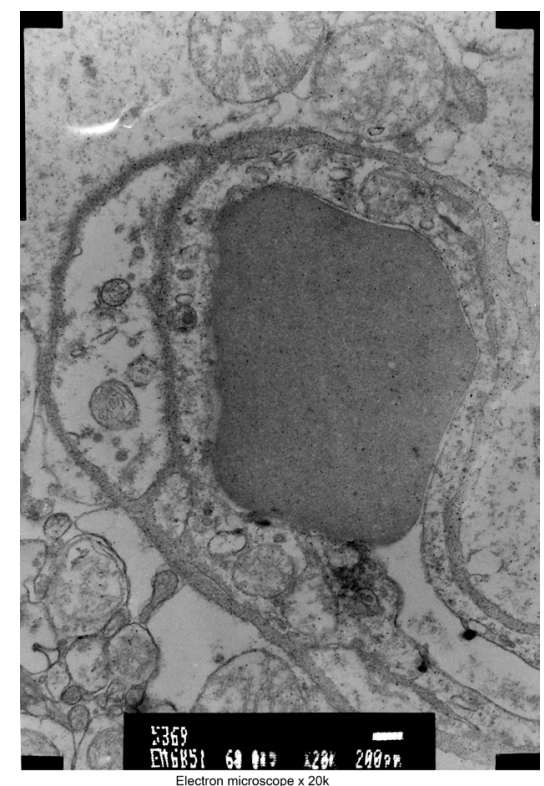

Figure 3. At the 12th month, the number of endothelial cells decreased and the basement membrane thickened and became uneven. The tight junctions between cells disappeared and organelles dissolved and disappeared.

\section{Correlation between rCBV and VEGF}

According to Pearson product moment correlation analysis, $r=0.94(P<0.05)$. There was a significant and positive linear correlation between $\mathrm{rCBV}$ and VEGF.

\section{DISCUSSION}

\section{Index of MRP and immunohistochemistry detection}

MRP is one of the most useful methods for diagnosing radiation brain injury. As a functional imaging technology, this method can reflect the blood perfusion status in brain tissues. Common indices used in MRP include rCBV, relative cerebral brain flow, MTT, and TTP. The value of rCBV is closely related to microvessel density, while decreased rCBV represents decreased vessel density and tissue perfusion; relative cerebral brain flow reflected blood flow in the brain tissue; MTT reflected the time required for the contrast agent to flow through the capillary; TPP was the time required for the blood to flow through a specific volume of brain tissue (Cha et al., 2002; Cha et al., 2002). Among these factors, rCBV reflected blood perfusion and is most commonly used in the clinical setting.

VEGF is the strongest cytokine promoting angiogenesis, exists in many tissues in human and animals, and plays a role in physiological regulation (Harada et al., 1994; Park et al., 1994). Our results showed that VEGF expression was closely related to microvessel density and microvasculature density. After radiation brain injury, VEGF expression and the microvessel network were altered. Currently, VEGF is the reliable marker for observing microcirculation changes in studies of radiation brain injury. The absorbance value of VEGF can be determined using a microscope camera system (Wang et al., 2006). 


\section{Correlation between MRP and pathology}

Numerous studies have examined radiation brain injury using MRP. Nearly all of the results have shown that $\mathrm{rCBV}$ at the damage field is decreased and the values of MTT and TTP were prolonged. Bai et al. (2014) studied the effect of blood perfusion of radiation brain injury and found that most rCBV of lesions decreased compared with in the host contralateral cortex. Zhao et al. (2005) found that rCBV in the edema area of delayed radiation brain injury was decreased, while MTT and TTP were prolonged. Zhang et al. (2009) showed that during the late stage of radiation, microcirculation arterioles showed fibrinoid necrosis and the vascular wall was infiltrated by numerous plasma proteins and presented vitreous degeneration. This induced basement membrane thickening and lumen narrowing or blockage, leading to a decrease in rCBV.

Our results were consistent with those of previous studies. rCBV decreased gradually while MTT and TTP increased in the radiation groups, and the absorbance value of VEGF decreased slightly. Vascular density and structure were important factors influencing cerebral tissue perfusion, while the changes of the two induced changes in cerebral tissue perfusion. Based on the results of light microscopy, beginning in the 3rd month after radiation, vascular density in the brain tissue decreased slightly, the vascular wall thickened, and the lumen became narrower or blocked. According to the results of electron microscopy, beginning in the 3th month after radiation, endothelial cells in the brain tissue underwent apoptosis and the basement membrane thickened. Through the 12th month, the number of endothelial cells showed a large decrease, the basement membrane clearly thickened, the lumen became narrower, and organelle structures dissolved and disappeared. At the region of radiation brain injury, pathological changes that can be detected using MRP include decreased vascular density, narrowing lumen, decreased number of endothelial cells, and basement membrane thickening. Blood vessel damage caused a reduction in the blood supply at the region of radiation. Blood perfusion in the brain tissue was decreased, as revealed by the decreased in rCBV and increase in MTT and TTP. The absorbance value of VEGF in the radiation groups was slightly decreased, which was positively correlated to $\mathrm{rCBV}$. These results indicate that VEGF is a key factor leading to changes in the macrostructure and microstructure of brain vessels in the region of radiation. The decrease in VEGF led to decreased vessel density and a decreased number of endothelial cells. The vessel decrease led to $\mathrm{rCBC}$ at the region of radiation decrease. Therefore, decreased VEGF likely plays an important role in the pathogenesis of brain radiation injury.

In conclusion, MRP reflects the pathological changes in radiation brain injury, which can be used for early diagnosis; decreased VEGF expression is the pathological basis of brain radiation injury, and this decreased expression can be detected using MRP.

\section{Conflicts of interest}

The authors declare no conflict of interest.

\section{ACKNOWLEDGMENTS}

Research supported by the People's Livelihood Project in Science and Technology Development Plan of Qingdao (Grant\#14-2-3-7-nsh) and the Science and Technology Development Plan of Qingdao (Grant \#12-1-4-2-(10)-jch). 


\section{REFERENCES}

Asai A and Kawamoto K (2008). Radiation-induced brain injury. Brain Nerve 60: 123-129.

Bai XF, Niu GM, Han XD, Gao Y, et al. (2014). Differentiation between recurrent glioma and radiation-induced brain injuries using perfusion weighted imaging and diffusion weighted imaging. Chin. J Mag. Res. Imag. 5: 7-10.

Cha S, Knopp EA, Johnson G, Wetzel SG, et al. (2002). Intracranial mass lesion: dynamic contrast-enhanced susceptibilityweighted echo-planar perfusion MR imaging. Radiology 223: 11-29.

Harada S, Nagy JA, Sallivan KA, Thomas KA, et al. (1994). Induction of vascular endothelial growth factor expression by prostaglandin E2 and E1 in osteoblasts. J. Clin. Invest. 93: 2490-2496.

Park JE, Chen HH, Winer J, Houck KA, et al. (1994). Placenta growth factor potentiation of vascular endothelial growth, in vitro and vivo, and high affinity binding to Flt-1 but not to Flk-1/KDR. J. Biol. Chem. 269: 25646-25654.

Tsao MN, Lloyd NS, Wong RK, Rakovitch E, et al. (2005). Radiotherapeutic management of brain metastases: a systematic review and meta-analysis. Cancer Treat. Rev. 31: 256-273.

Wang C, Tian Y, Xu FQ, Xie H (2006). The study on changes of the blood-brain barrier after radiation-induced brain injury in the early stage in rats. Chin. J. Hemorheol. 16: 325-328.

Zhang FS, Xiao SH, Liu J, Liu YL, et al. (2009). The treatment of mesenchymal stem cells transplantation combining with vascular endothelial growth factor gene can improve radiation cognitive impairment. Chin. J. Beh. Med. Brain Sci. 18: 25-27.

Zhao JQ, Liang BL, Shen J, Zhang XH, et al. (2005). Magnetic resonance perfusion weighted imaging manifestation of late radiation-induced encephalopathy at temporal lobe. Ai Zheng 24: 1102-1105. 